\title{
Systematic Safety Evaluation of Cold Plasma-Activated Liquid in Rabbits
}

\author{
Yujing $X u^{1}$, Sansan Peng ${ }^{1}$, Bing $L i^{2}$, Shuai Wang ${ }^{2}$, Hao Zhang ${ }^{1}$, Qiaosong $L i^{1}$, Zhijie Liu ${ }^{1}$, \\ Bo Guo ${ }^{1}$, Dingxin $\mathrm{Liu}^{1 *}$ and Dehui $\mathrm{Xu}^{1 *}$ \\ ${ }^{1}$ State Key Laboratory of Electrical Insulation and Power Equipment, Centre for Plasma Biomedicine, Xi'an Jiaotong University, \\ Xi'an, China, ${ }^{2}$ The School of Life Science and Technology, Xi'an Jiaotong University, X'an, China
}

OPEN ACCESS

Edited by:

Mounir Laroussi, Old Dominion University,

United States

Reviewed by:

Hiromasa Tanaka,

Nagoya University, Japan

Alan A. Howling,

École Polytechnique Fédérale de

Lausanne, Switzerland

Lenka Zajičková,

Masaryk University, Czechia

*Correspondence:

Dingxin Liu

liudingxin@mail.xjtu.edu.cn

Dehui Xu

dehuixu@mail.xjtu.edu.cn

$$
\begin{array}{r}
\text { Specialty section: } \\
\text { This article was submitted to } \\
\text { Plasma Physics, } \\
\text { a section of the journal } \\
\text { Frontiers in Physics }
\end{array}
$$

Received: 27 January 2021 Accepted: 10 August 2021 Published: 24 August 2021

Citation: Xu Y, Peng S, Li B, Wang S, Zhang $H$,

Li Q, LiU Z, Guo B, LiU D and Xu D (2021) Systematic Safety Evaluation of Cold Plasma-Activated Liquid in Rabbits.

Front. Phys. 9:659227. doi: 10.3389/fphy.2021.659227
Plasma-activated liquid (PAL) can effectively and selectively kill various types of cancer cells both in superficial and deeper tumors. As a promising novel approach to oncotherapy, the safety of PAL is essential in the clinic but has not been thoroughly assessed. In myeloma and blood tumors, the pathogenesis is in the bone marrow cavity. We have therefore evaluated the safety of PAL in New Zealand rabbits by intra-bone marrow injection, and provide a basis of further clinical research and application of PALs. In this study, both a plasma jet and plasma surface were used to treat saline solution, phosphate-buffered solution, and cell culture medium, to produce PAL. Then, oscillograms and optical emission spectra were evaluated to characterize the plasma discharge. Acute toxicity tests and safety evaluation studies were conducted by intra-bone marrow injection of PAL into New Zealand rabbits, while control rabbits received saline only. Body weight, vital organ coefficient, organ appearance, organ histopathology, blood cell and hemoglobin parameters, and blood biochemical indicators were tested on the 30th day after injection. We found that there was no mortality or loss of mobility throughout the experimental period. Acute toxicity tests showed that there were no PAL-related side effects in rabbits receiving the maximum dose of $700 \mu \mathrm{L}$ PAL. PAL treatments did not affect body weight, organ coefficient, organ appearance, organ histopathology, or blood biochemical indicators. However, the percentage of lymphocytes decreased while the percentage of neutrophil granulocytes increased compared with the control group. In summary, our results indicate that PAL can be safely injected into bone marrow of New Zealand rabbits without significant toxicity.

Keywords: cold atmospheric plasma, plasma-activated liquid, New Zealand rabbits, safety evaluation, tumor

\section{INTRODUCTION}

The number of cancer cases is increasing in both developed and developing countries, and cancer continues to be a major health problem that has in recent years placed a heavy burden on families and society. According to GLOBOCAN2020 statistics, patients in China account for approximately $23.7 \%$ of fresh cancer cases and $30 \%$ of malignant tumor-related deaths worldwide [1]. Accumulating evidence has confirmed that cancer relapse and drug resistance are problems that urgently need to be addressed, despite great progress having been made in the field of cancer therapy [2, 3]; thus it is necessary to introduce new therapeutic strategies for cancer treatment.

In this study, we focused on the emerging technology of cold atmospheric plasma (CAP). Plasma is defined as a fourth state of matter in addition to solid, liquid, and gas. Thermal and non-thermal 
plasma are ionized gases composed of electrons and ions [4]. CAP is a typical non-thermal plasma, and since it can be produced at atmospheric pressure and near room temperature, it has been used in many medical fields, including wound healing, dentistry, sterilization, dermatology, and oncology, among others [5-8]. Many studies have shown that CAP can efficiently kill various types of tumor cells, including melanoma cells, colorectal cancer, leukemia cancer, multiple myeloma, and others [8-12]. However, the underlying mechanisms by which tumor cells sustain damage remain unclear. Many scholars have proposed that reactive oxygen species (ROS) and reactive nitrogen species (RNS) generated by CAP might play a significant role in the induction of cancer cell death $[13,14]$.

Depending on its application to biological tissues, CAP can be classified as direct or indirect plasma. Direct plasma, which employs direct application of the CAP device, is more suitable for treating surface diseases like cutaneous carcinoma and wound healing $[5,8]$. Nevertheless, penetration of direct plasma into tissue is limited, and results from simulations showed that the active substances produced by plasma only extend into skin or tissues by $1-3 \mathrm{~mm}$ [15], which limits its use in clinical applications. Development of indirect plasma addressed this limitation. Indirect plasma, or plasma-activated liquid (PAL), involves treating a liquid with CAP, and the liquid is then applied to cells. Notably, PAL maintains its biological activity for at least 7 days when stored at $-80^{\circ} \mathrm{C}$ or $4^{\circ} \mathrm{C}[16,17]$, allowing it to be prepared in advance and later applied to cancer cells and tissues without a CAP device. Moreover, PAL can be used in solid and blood tumors that are not near the surface of the body by injecting it into tumor sites deep in tissue. Previous investigations showed that PAL could inhibit the growth of leukemia, bone cancer, pancreatic adenocarcinoma cells, glioblastoma cells, and other cancer cells in vitro $[13,18,19]$. Canal et al. [19] also found that the cytotoxicity of PAL indirect treatment of tumor cells was comparable to that of CAP direct treatment. Consequently, PAL greatly expands the potential application and development of CAP in medicine.

However, the biosafety of PAL in animals has not been systematically studied. Biscop et al. [20] reported that various components of plasma-treated liquid greatly affect cellular cytotoxicity of PAL. Thus, we selected three kinds of liquid in this study-saline, phosphate buffer solution (PBS), and cell culture medium-to evaluate the safety of different PALs in New Zealand rabbits by intra-bone marrow injection, and to provide further support for the safe application of PALs in the clinic.

\section{EXPERIMENTAL SETUP AND METHODS}

\section{Plasma Generation Systems}

The plasma jet system consisted of a gas flow controller, highvoltage power, oscilloscope, and plasma device. The plasma jet with a gas flow of two slm $\mathrm{He}$ (standard liters per minute with helium as the carrier gas) and an additional $0.01 \mathrm{slm}$ of $\mathrm{O}_{2}$ was excited by a power supply generating a peak-to-peak sinusoidal voltage of $8 \mathrm{kV}$ at a frequency of $10 \mathrm{kHz}$ to generate $\mathrm{He}+\mathrm{O}_{2}$ plasma. The jet length can be up to $1.5-2 \mathrm{~cm}$. Figures $\mathbf{1 A}, \mathbf{B}$ shows a diagram of the plasma jet device and a photograph of the jet itself. The plasma jet source adopted a needle-to-ring electrode structure. And a stainless-steel needle with an inside diameter of $1 \mathrm{~mm}$ was used as a high voltage electrode, which was enclosed in a $6 \mathrm{~cm}$ long quartz tube and about $15 \mathrm{~mm}$ above the top of the quartz tube. Quartz glass tubes with outer diameter of $6 \mathrm{~mm}$ and inner diameter of $4 \mathrm{~mm}$ were used as the insulating medium. A $10 \mathrm{~mm}$ copper ring was placed around the wall of the quartz tube as the ground electrode, $10 \mathrm{~mm}$ away from the tube at the bottom.

The plasma surface was generated from ambient air using the same power as that applied to the plasma jet device. Figures 1C,D shows a diagram of the device and image of the discharge. The air surface discharge device is a sandwich structure formed by a dielectric layer and two electrodes. The dielectric layer is sandwiched between a high-voltage electrode and a mesh ground electrode. Each mesh element has a hexagonal shape.

The images were captured by a digital camera (Nikon D7200) using an exposure time of $0.5 \mathrm{~s}$. We used an oscilloscope (Tektronix, DPO3000) to record the waveform of the discharge voltage and current detected by a high-voltage probe (Tektronix, P6015A) and a current probe (Tektronix, P6021), respectively (Figures 2A,B). It can be seen that for both devices, the positive and negative half-cycle current waveforms were asymmetrical. When the same voltage was applied at $8 \mathrm{kV}$, the jet device current generated by a positive half period voltage consisting of a series of small pulses typical of glow discharge. For the surface device, the peak current appeared at the rising and falling edges of the voltage waveform, which was composed of a series of small pulses. This is typical of filamentous discharge. Power waveforms of the jet and surface devices are plotted in Figures 2C,D, respectively. Average power was calculated by integrating voltage and current across multiple discharge cycles. The average discharge power of the jet device was $1.2 \mathrm{~W}$, while that of the surface device was $4.62 \mathrm{~W}$.

\section{Measurements of Physicochemical Properties of PALs}

Plasma discharge was used to treat three types of liquid: strokephysiological saline solution $(0.9 \% \mathrm{NaCl}$; SMARBO, Ningbo Yinuo Medical Equipment Co., Ltd., China), $1 \times$ PBS (Thermo Fisher Scientific, MA, United States; Cat No. 10010002), and RPMI (Roswell Park Memorial Institute) 1,640 complete medium containing $10 \%$ fetal bovine serum and $1 \%$ penicillin-streptomycin solution (Corning, NY, United States; Cat No.10-040-CVRC). For plasma jet treatment, $300 \mu \mathrm{L}$ of liquid were placed in a 24 -well plate and treated for $3 \mathrm{~min}$, with a $10 \mathrm{~mm}$ distance between the end of plasma jet plume and the liquid surface. The three solutions treated by plasma jet discharge were $0.9 \% \mathrm{NaCl}, 1,640$ medium, and $1 \times \mathrm{PBS}$, and these treatment groups were abbreviated J-N, J-M, and J-P, respectively. For plasma surface treatment, $3.5 \mathrm{ml}$ of liquid were placed in a $60 \mathrm{~mm}$ diameter dish $10 \mathrm{~mm}$ away from the plasma surface device and treated for $3 \mathrm{~min}$. The same three solutions were treated by plasma-surface discharge, and the groups were abbreviated S-N, S-M, and S-P.

The $\mathrm{pH}$ and oxidation-reduction potential (ORP) of the liquids were measured with a $\mathrm{pH}$ meter (METTLER-TOLEDO, FE20) and a 

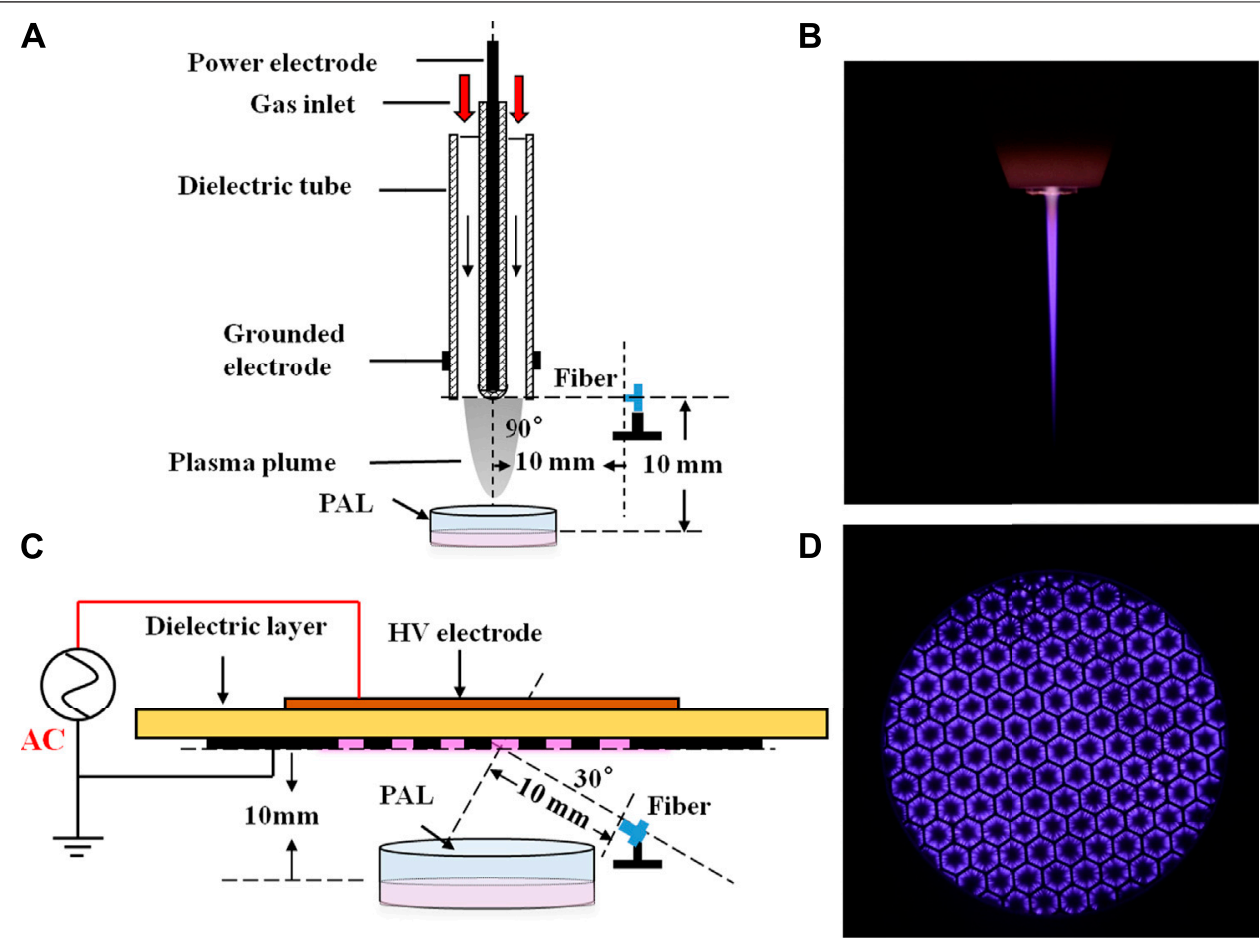

FIGURE 1 | Low temperature plasma jet/surface at atmospheric pressure. (A) Structure and (B) photograph of the plasma jet device; (C) structure and (D) photograph of the plasma surface device.

benchtop ORP meter (METTLER-TOLEDO, S210-K), respectively. The concentration of aqueous long-lived reactive oxygen and nitrogen species (RONS) of PALs induced by the plasma jet and surface devices was measured as follows. $\mathrm{H}_{2} \mathrm{O}_{2}$ was detected using a hydrogen peroxide assay kit (Beyotime, Shanghai, China; Cat No. S0038), $\mathrm{NO}_{2}^{-}$was detected using a nitric oxide assay kit (Beyotime, Shanghai, China; Cat No. S0024), and absorbance was measured using a microplate analyzer (Thermo Science, MA, United States).

\section{Optical Emission Spectroscopy}

Optical emission spectroscopy (OES) of plasma jets and surfaces was performed using an Andor SR-750i grating monochromator (grating grooving 1,200 lines $\mathrm{mm}^{-1}$ ) in the presence of liquid. Wavelength ranged from 200 to $800 \mathrm{~nm}$. The locations of the optical probe in the two devices are shown in Figures 1A,C. Light emitted by the plasma jet was collected at the nozzle of the quartz tube by an optical fiber perpendicular to the center of a quartz tube, which was focused on the jet plume at a distance of $1 \mathrm{~cm}$ from the discharge area. For the surface device, the optical probe was $30^{\circ}$ from the horizontal at a distance of $1 \mathrm{~cm}$ from the discharge area. When measuring the spectra of different liquids treated by the two devices, OES detected a discharge area of about $2 \mathrm{~mm}^{2}$.

\section{Animals and PAL Treatment}

Healthy New Zealand Rabbits, weighing $2.35 \pm 0.35 \mathrm{~kg}(p>0.05)$, were raised in the Experimental Animal Center of Xi'an Jiaotong University under an experimental animal production license [SCXK (shaan) 2008-008] and animal use certificate [SYXK (shaan) 2008-
002] issued by the Science and Technology department of Shaanxi province. They were housed individually in clean cages and were given water and a diet of pellets ad libitum. The animal house was maintained at a room temperature of about $20^{\circ} \mathrm{C}$ and $60 \%$ relative humidity. Twenty-one rabbits were randomly divided into seven groups of three rabbits each. Before treatment, all rabbits were anesthetized with sodium pentobarbital by slow injection via an ear side vein. Then, the surgical area was sterilized with iodine after gently removing hair. The puncture needles were used to inject the preparations at $0.3-0.5 \mathrm{~cm}$ below the tip of the third trochanter of the femur. Each of the rabbits was given $0.4 \mathrm{ml}$ of prepared PAL and an equal volume of physiological solution was given to the control rabbits. After surgery, cefuroxime sodium was injected to reduce inflammation. Acute toxicity experiments in response to injection of PAL into bone marrow were performed at a dose of $700 \mu \mathrm{L}$ saline using the above surgical procedures. During the experimental period ( 30 days post-injection), the rabbits were observed daily for any adverse effects such as illness or death, including changes in mental, dietary, and diarrhea status. After fasting overnight, all animals were sacrificed to collect blood and organ samples.

\section{Analysis of Blood Biochemical Index and Blood Cell}

At 8-9 am 30 days after surgery, blood from each rabbit was drawn from an ear vein. Then $1.5 \mathrm{ml}$ of blood was injected into a vacuum anticoagulant tube containing EDTA that was gently 

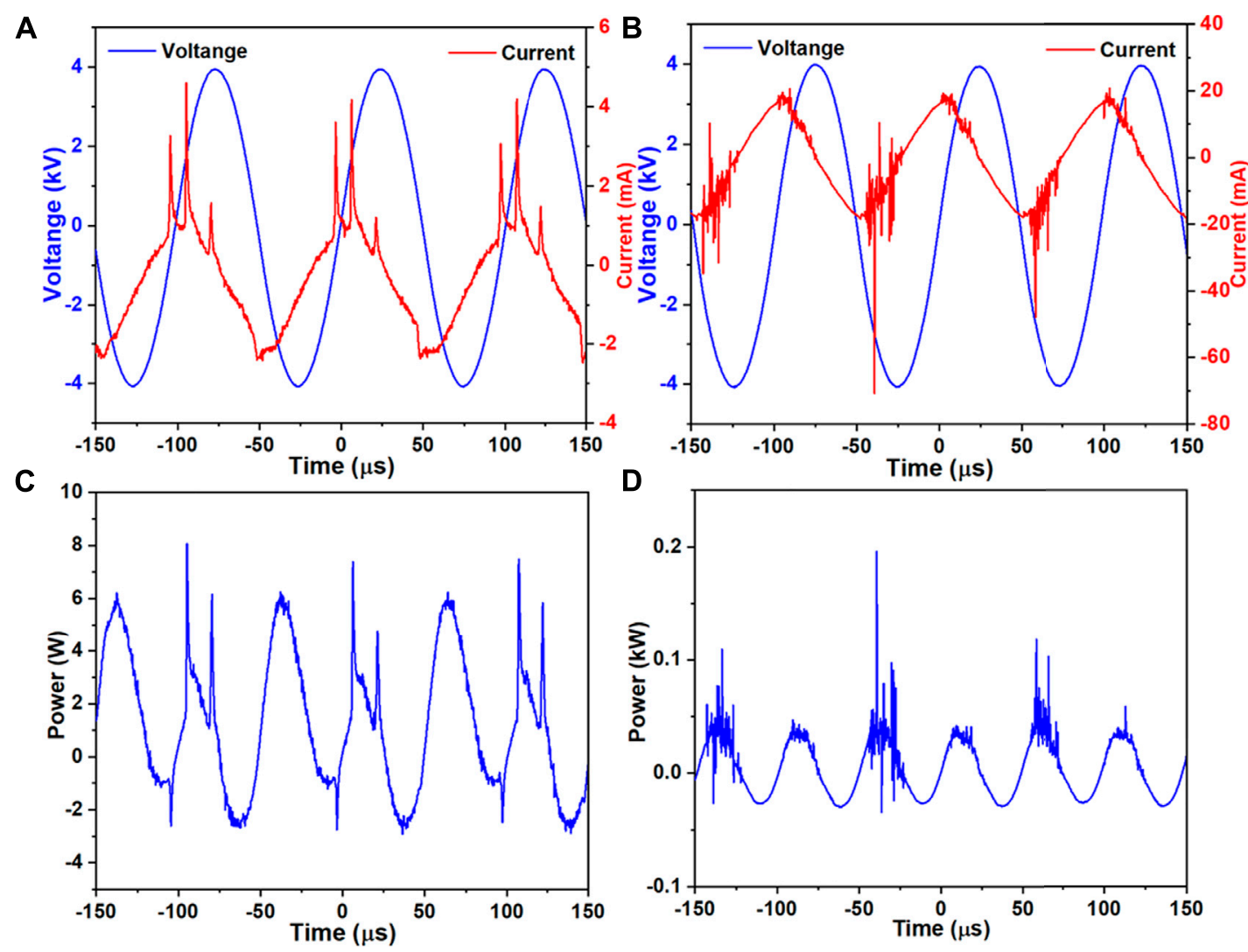

FIGURE 2 | Waveforms of voltage and current (A, B) and power (C, D) for the jet (A, C) and surface (B, D) devices.

inverted several times to mix the anticoagulant and blood. Whole blood was used for routine blood testing, which was performed by an automatic blood cell analyzer (Mindray veterinary automatic blood cell analyzer; model: BC-2800vet). The remaining $3.5 \mathrm{ml}$ of blood was injected into a $15 \mathrm{ml} \mathrm{EP}$ tube, which was placed on a table at a tilt of $30^{\circ}$ for $30 \mathrm{~min}$. Serum, used to test liver and kidney function and other biochemical indicators, was obtained by centrifugation of the whole blood at 3,000 rpm for $10 \mathrm{~min}$. All biochemical indexes were tested by an automatic biochemical analyzer (Shenzhen Rayto life science co. Ltd.; model: Chemray 240).

\section{Coefficients of Vital Organs}

The rabbits were weighed on day 30 and sacrificed after blood was taken and the abdomen and chest were opened with a scalpel to assess the organs. The connective tissue around the heart, liver, spleen, kidney, and lung was cut away and these organs were excised and weighed. The coefficient of an organ is expressed as organ index = weight of the organ $(\mathrm{g}) /$ weight of the rabbit (g) $\times 100 \%$.

\section{Histopathological Examination}

After photographing each organ, small pieces of tissues from the same part of the same organ (heart, liver, lung, and kidney) were put into $4 \%$ paraformaldehyde, which was prepared in advance, and fixed at $4^{\circ} \mathrm{C}$ for $12 \mathrm{~h}$. Subsequently, they were dehydrated in
$30 \%$ sucrose and then embedded in OCT (optimal cutting temperature) compound. The tissue specimens were sliced into $5 \mu \mathrm{m}$ sections with a freezing microtome (Leica CM1950, Leica Biosystems Nussloch GmbH, Germany) and then stained with hematoxylin and eosin (HE), according to standard protocols. After that, tissue slides were processed for histopathological examination under an optical microscope (BX53 and DP73, Olympus, Tokyo, Japan).

\section{Statistical Analysis}

In this study, SPSS 23 statistics software was used for statistical analysis. All data are expressed as mean \pm standard error of the mean (SEM) for three independent experiments and analyzed by an independent sample $t$-test and one-way analysis of variance (ANOVA), followed by post-hoc multiple comparisons tests such as LSD and Duncan's test. Differences were considered statistically significant when the $p$ value was less than or equal to 0.05 ( $p \leq 0.05)$ with a confidence interval of $95 \%$.

\section{RESULTS}

\section{Physicochemical Properties and Long-Lived RONS of PALs}

As shown in Figures 3A,B, there were only small differences in the $\mathrm{pH}$ and $\mathrm{ORP}$ of the $0.9 \% \mathrm{NaCl}$ solution, $\mathrm{PBS}$ and cell culture 

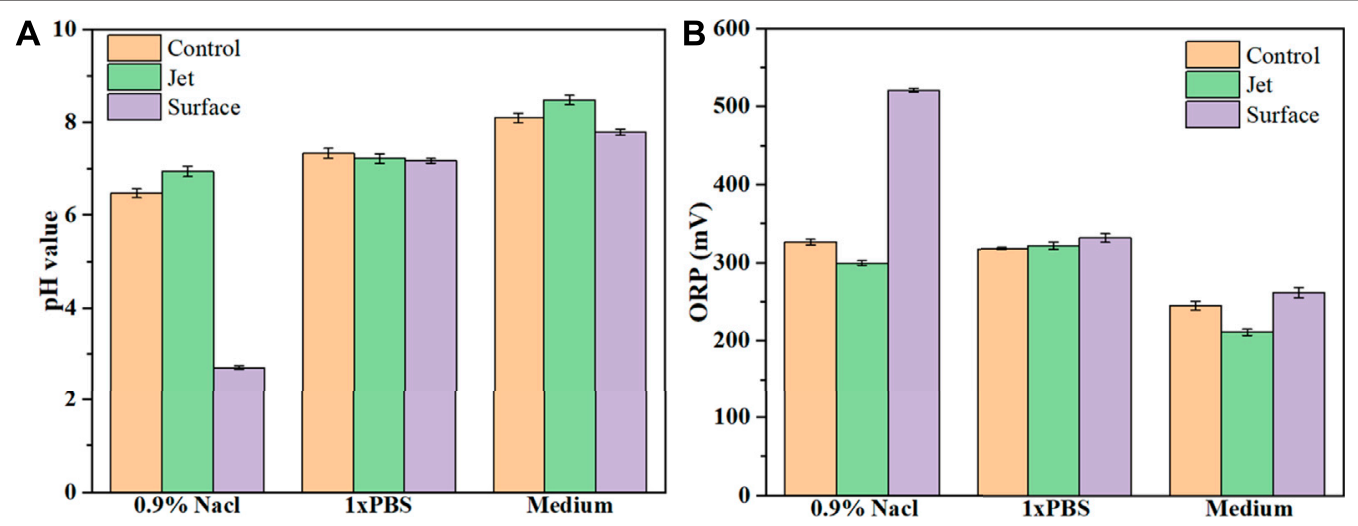

FIGURE 3 | Values of $\mathrm{pH}$ (A) and ORP (B) in control, plasma jet- and surface-treated $0.9 \% \mathrm{NaCl}$ solution, PBS, and 1,640 medium. Solutions were treated by the plasma jet or surface devices for 3 min.
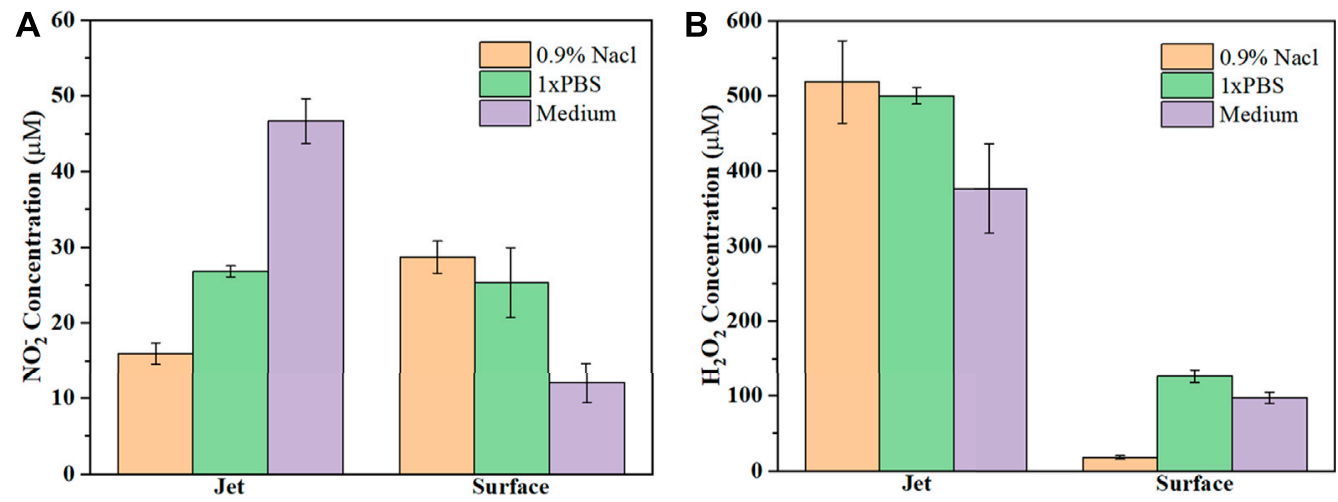

FIGURE 4 | Concentration of (A) $\mathrm{NO}_{2}^{-}$and (B) $\mathrm{H}_{2} \mathrm{O}_{2}$ in the three types of PALs. All solutions are treated by the plasma jet or surface device for 3 min

medium for the control group (no plasma treatment), mainly due to different liquid components. When comparing the control and plasma jet groups, we found that $\mathrm{pH}$ and ORP in PBS was little affected by jet treatment, however, the $\mathrm{pH}$ of the $0.9 \% \mathrm{NaCl}$ solution and culture medium increased slightly while ORP exhibited a downward trend after jet treatment. In the case of the plasma surface device, the $\mathrm{pH}$ of all three solutions decreased when compared with the control and plasma jet groups. In contrast, we found that plasma surface treatment reduced the $\mathrm{pH}$ of the $0.9 \% \mathrm{NaCl}$ solution much more than PBS and culture medium. ORP in PALs exposed to plasma surface treatment was negatively correlated with $\mathrm{pH}$. Figures 4A,B shows the concentration of $\mathrm{H}_{2} \mathrm{O}_{2}$ and $\mathrm{NO}_{2}{ }^{-}$in the PALs. We found that the $\mathrm{H}_{2} \mathrm{O}_{2}$ concentration produced by the jet device was more than twice as high as that produced by the surface device, while there was less difference in the generation of $\mathrm{NO}_{2}{ }^{-}$in the liquids exposed to these two devices.

\section{Spectra Characteristics}

OES was used to determine the excited products of plasma treatment. Emission spectra were measured for treatment of PBS, $0.9 \% \mathrm{NaCl}$ solution and RPMI 1640 medium by the $\mathrm{He}+0.5 \% \mathrm{O}_{2}$ plasma jet (Figures 5A-C, respectively) and the plasma surface device (Figures 5D-F, respectively). For both the plasma jet and surface devices, the spectral bands were the same regardless of the solution being treated, but the intensity of each band varied slightly. However, the plasma jet and surface devices had quite different emission spectra. The spectrum of the plasma surface device was mainly composed of the first negative band of $\mathrm{N}_{2}^{+}\left(\mathrm{B}_{2} \Sigma \mathrm{u}^{+} \rightarrow \mathrm{X}_{2} \Sigma \mathrm{g}^{+}\right)$, the second positive band of $\mathrm{N}_{2}\left(\mathrm{C}_{3} \Pi \mathrm{u} \rightarrow \mathrm{B}_{3} \Pi g\right)$, and the second order diffraction of $\mathrm{N}_{2}\left(\mathrm{C}_{3} \Pi \mathrm{u} \rightarrow \mathrm{B}_{3} \Pi \mathrm{g}\right)$. In addition to the first negative band of $\mathrm{N}_{2}{ }^{+}\left(\mathrm{B}_{2} \Sigma \mathrm{u}+\rightarrow \mathrm{X} 2 \Sigma \mathrm{g}+\right)$ and the second positive band of $\mathrm{N} 2(\mathrm{C} 3 \Pi \mathrm{u} \rightarrow \mathrm{B} 3 \Pi \mathrm{g})$, the spectrum of the plasma jet device also contained the band of $\mathrm{OH}$ radicals and some excited states of $\mathrm{He}$.

\section{Acute Toxicity Test by Different PAL Injections}

Acute toxicity experiments were conducted by injecting PAL into the bone marrow of rabbits. As shown in Table 1, no rabbit died after injection of $700 \mu \mathrm{L}$ plasma jet- or plasma surface-treated PAL. We continuously observed the rabbits for one month after 

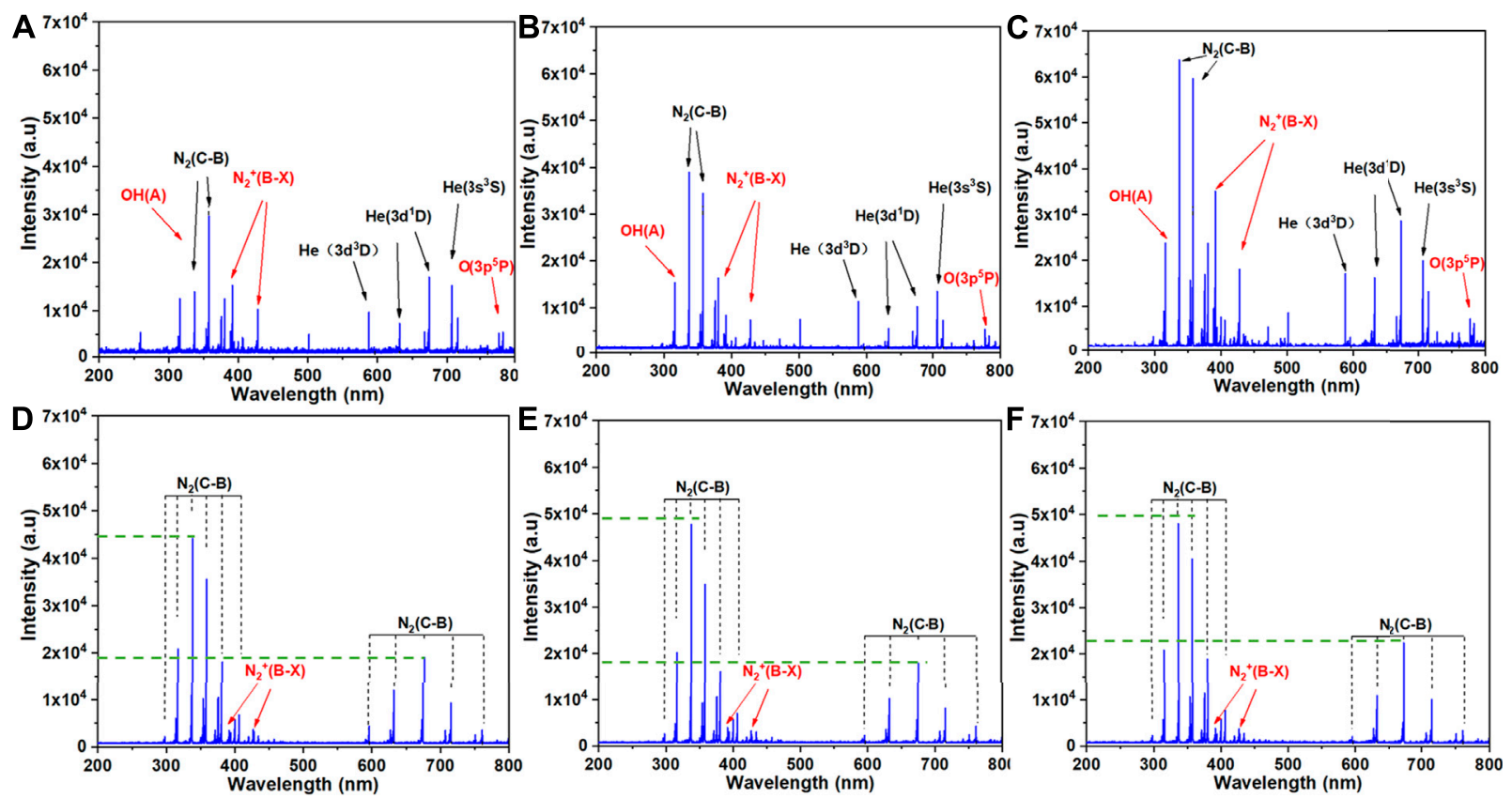

FIGURE 5 | Emission spectra of the two plasma discharge devices in the presence of the three types of solution: (A) J-P, (B) J-N, (C) J-M, (D) S-P, (E) S-N and (F) S-M. The six treatment groups are described in Experimental setup and methods.

TABLE 1 | Acute toxicity tests of PAL treatments of New Zealand rabbits.

\begin{tabular}{|c|c|c|c|c|c|c|}
\hline Treatment & $\begin{array}{c}\text { Plasma dose } \\
(\mu L)\end{array}$ & Rabbit numbers & $\begin{array}{l}\text { Initial body } \\
\text { weight (kg) }\end{array}$ & $\begin{array}{l}\text { Final body } \\
\text { weight (kg) }\end{array}$ & Deaths & MTD $(\mu L)$ \\
\hline Jet & 700 & 6 & $2.5 \pm 0.2$ & $3.0 \pm 0.3$ & 0 & $>700$ \\
\hline Surface & 700 & 6 & $2.5 \pm 0.3$ & $3.0 \pm 0.3$ & 0 & $>700$ \\
\hline
\end{tabular}

PAL injection. For either plasma jet- or plasma surface-treated PALs, body weight of the rabbits after PAL injection was similar to that of the control group $(p>0.05)$. The rabbits showed no abnormal behavior, such as mental state, excretion, food intake, etc. Therefore, the maximum tolerated dose (MTD) of PAL injection in the bone marrow of rabbits is greater than $700 \mu \mathrm{L}$.

\section{Changes of Body Weight and Vital Organs After PAL Injection}

Throughout the experimental period, no abnormal behavior or symptoms were observed in the rabbits. To evaluate the effect of different PALs, body weight and several vital organs were assessed 30 days after injection. There was no significant change in body weight between groups ( $p>0.05$; Figure 6A). After sacrificing the rabbits, organ coefficients were calculated and are shown in Figure 6B. No obvious differences in cardiac index, liver index, spleen index, lung index, or kidney index were found among the groups $(p>0.05)$.

Furthermore, post-mortem observations revealed that the heart, liver, spleen, lung, and kidneys of the rabbits in each treatment group had a shiny surface, uniform color, and a compact and flexible texture, as shown in Figure $6 \mathrm{C}$, indicating that no abnormal organ morphology and no obvious macroscopic lesions emerged after different PAL treatments. Additionally, it can be seen in the images of Figure 6C that there were no visible differences in size, color, appearance, or morphology of each organ between the control group and the different PAL treatment groups.

\section{Histopathological Changes in Vital Organs After PAL Injection}

To assess the effect of the different PALs on microscopic tissue structure, frozen sections of the heart, liver, lung, and kidneys were processed for $\mathrm{HE}$ staining and visualization by light microscopy. Figure 7shows that there were no unusual pathological changes in the heart, liver, lung, and kidney tissue sections of the rabbits in each treatment group.

The images of heart tissue from each group showed that heart structure was intact and myocardial cells were cylindrical. The myocardial fibers were neatly arranged with clear horizontal lines, and no obvious atrophy, degeneration, hypertrophy, or necrosis was observed. In addition, no abnormalities, such as hemorrhage, 

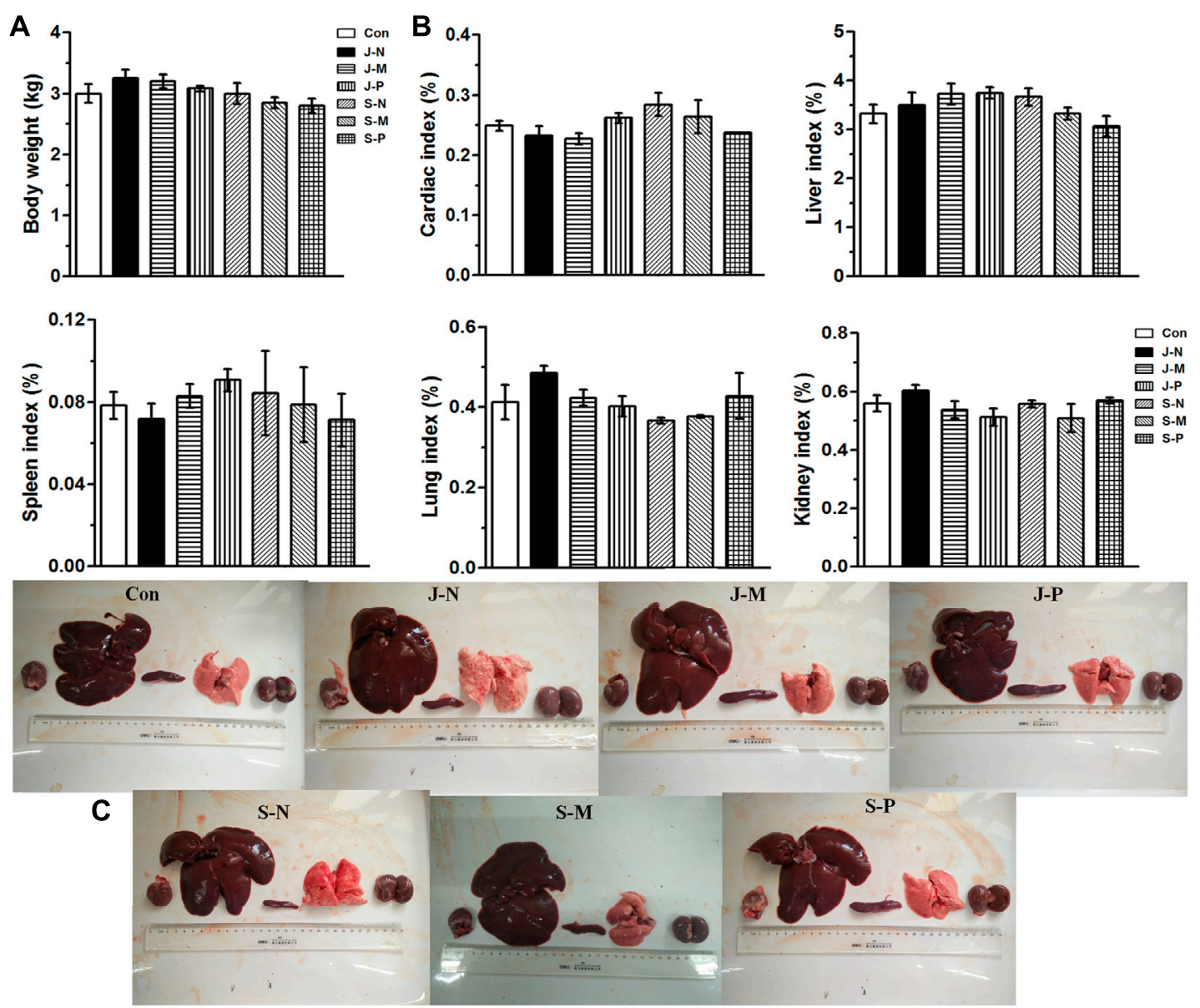

FIGURE 6|Effects of different PALs at 30 days after injection on (A) body weight; (B) organ coefficient, including cardiac index, liver index, spleen index, lung index, and kidney index; (C) photographs of vital organs (heart, liver, spleen, lungs, and kidneys) in New Zealand rabbits ( $n=3$ rabbits/group, mean \pm SEM). The control and six treatment groups (Con, J-N, J-M, J-P, S-N, S-M, and S-P) are as described in Experimental setup and methods.

were observed in the myocardial interstitium. This illustrated that there was no damage to the heart tissue after different PAL treatments and there were no significant differences when compared with the control group (Figure 7).

Liver structure appeared normal, with the central vein, hepatic sinus, hepatic lobule, and portal area clearly visible. Moreover, hepatocytes were arranged tightly and tidily, with the intermediate nucleus and the morphology being clear. No abnormalities, such as necrosis and degeneration, were seen in the liver cells. The liver sections showed no hyperplasia of collagen fibers in the central vein or perisinusoidal space, as well as no infiltration of the inflammatory cells or proliferation of connective tissue in the manifold area after different PAL treatments and no visible difference when compared to the control group (Figure 7).

The lung tissue structure was complete and clear, and the lung cells were regularly arranged without inflammatory cell infiltration. The alveoli were uniform in size, and no inflammatory exudates or thrombus were seen in the alveolar cavity or the capillaries of the alveolar wall, respectively. The epithelium of all the levels of bronchioles was intact, and no deformation or dilatation of each bronchiole, as well as no necrosis or degeneration of each bronchiole epithelium, was observed. No pulmonary interstitial inflammation, fibrosis, pulmonary edema, alveolar hemorrhage, emphysema, hyaline membrane formation, alveolar epithelial hyperplasia, etc., occurred after different PAL treatments and there was no difference when compared to the control group.

The structure of the renal tissue was complete and the epithelial cells of the renal tubules were arranged regularly, and the renal cortex, medulla, and nephrons were visible and had normal structure. There was no abnormal change in the shape of the glomerulus and there were no obvious pathological changes in the proximal and distal renal convoluted tubules. Additionally, we observed no infiltration of inflammatory cells in the renal interstitium nor any infiltration of inflammatory cells, fibrinoid necrosis, or thrombosis into the blood vessels of the kidneys. Therefore, we observed no kidney injury in response to the different PAL treatments, and no apparent difference was seen between the PAL treatment and control groups.

\section{Changes of Blood Index After PAL Injection}

To check for toxic side effects of the different PAL injections, we assessed several blood parameters in the rabbits, including 


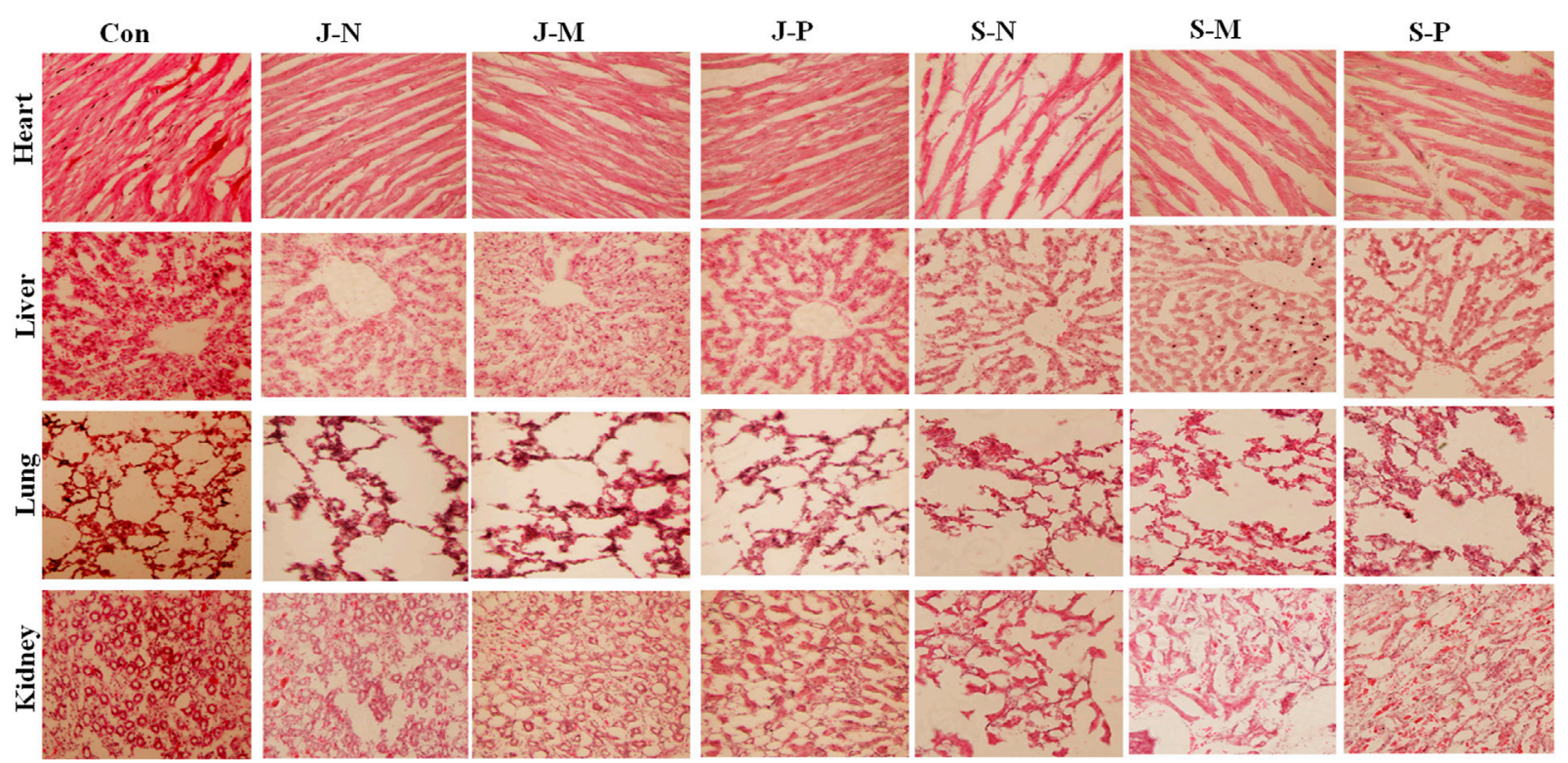

FIGURE 7| The histomorphological features of New Zealand rabbits after different PAL treatments. HE-stained images showed no pathological changes in heart, liver, lung, or kidney tissue when compared with the control group.

TABLE 2 | Effect of different PALs on blood cell and hemoglobin parameters.

\begin{tabular}{|c|c|c|c|c|c|c|c|c|}
\hline Parameter & Con & $\mathbf{J}-\mathbf{N}$ & $\mathbf{J}-\mathbf{M}$ & J-P & $\mathbf{S}-\mathbf{N}$ & S-M & S-P & $p$ Value \\
\hline WBC $\left(10^{9} / L\right)$ & $9.2 \pm 0.6$ & $10 \pm 1$ & $10 \pm 1$ & $11 \pm 2$ & $9.0 \pm 0.6$ & $10 \pm 1$ & $10 \pm 3$ & 0.985 \\
\hline Lymph $\left(10^{9} / \mathrm{L}\right)$ & $6.4 \pm 0.7$ & $7.5 \pm 0.7$ & $5 \pm 1$ & $5 \pm 1$ & $4.6 \pm 0.5$ & $4.3 \pm 0.9$ & $4 \pm 2$ & 0.215 \\
\hline Mon $\left(10^{9} / \mathrm{L}\right)$ & $0.30 \pm 0.06$ & $0.3 \pm 0.1$ & $0.23 \pm 0.09$ & $0.33 \pm 0.09$ & $0.27 \pm 0.03$ & $0.30 \pm 0.06$ & $0.23 \pm 0.09$ & 0.954 \\
\hline $\operatorname{Gran}\left(10^{9} / \mathrm{L}\right)$ & $2.6 \pm 0.2 b$ & $2.2 \pm 0.5 b$ & $4.2 \pm 0.6 \mathrm{ab}$ & $5.9 \pm 0.7 a$ & $4.2 \pm 0.2 \mathrm{ab}$ & $5.2 \pm 0.3 a$ & $6 \pm 1 a$ & 0.008 \\
\hline Lymph (\%) & $81.0 \pm 0.9 a$ & $70 \pm 10 a b$ & $60 \pm 20 a b c$ & $42 \pm 5 c$ & $51 \pm 2 b c$ & $43 \pm 5 c$ & $38 \pm 5 c$ & 0.011 \\
\hline Mon (\%) & $3.9 \pm 0.5$ & $6 \pm 3$ & $2.4 \pm 0.6$ & $3.3 \pm 0.7$ & $3.3 \pm 0.3$ & $2.8 \pm 0.3$ & $2.8 \pm 0.2$ & 0.497 \\
\hline Gran (\%) & $15 \pm 1 b$ & $22 \pm 7 b$ & $30 \pm 20 a b$ & $55 \pm 5 a$ & $46 \pm 2 a$ & $54 \pm 5 a$ & $59 \pm 5 a$ & 0.005 \\
\hline $\operatorname{RBC}\left(10^{12} / \mathrm{L}\right)$ & $5.5 \pm 0.1$ & $5.0 \pm 0.1$ & $5.5 \pm 0.7$ & $5.7 \pm 0.1$ & $6.4 \pm 0.2$ & $6.0 \pm 0.4$ & $5.8 \pm 0.5$ & 0.275 \\
\hline HGB $(g / L)$ & $103 \pm 2$ & $91 \pm 4$ & $100 \pm 10$ & $109.3 \pm 0.3$ & $118 \pm 7$ & $119 \pm 5$ & $108 \pm 5$ & 0.120 \\
\hline HCT (\%) & $40.7 \pm 0.4$ & $39 \pm 1$ & $40 \pm 4$ & $38.50 \pm 0.06$ & $42 \pm 2$ & $45 \pm 2$ & $40 \pm 2$ & 0.447 \\
\hline MCV (fL) & $74 \pm 1$ & $78.3 \pm 0.3$ & $73 \pm 3$ & $68 \pm 1$ & $69 \pm 3$ & $70 \pm 2$ & $69 \pm 5$ & 0.158 \\
\hline $\mathrm{MCH}(\mathrm{pg})$ & $18.7 \pm 0.5$ & $18.2 \pm 0.3$ & $19.0 \pm 0.9$ & $19.2 \pm 0.3$ & $18.3 \pm 0.5$ & $18.7 \pm 0.5$ & $19 \pm 1$ & 0.948 \\
\hline $\mathrm{MCHC}(\mathrm{g} / \mathrm{L})$ & $276 \pm 5$ & $287 \pm 7$ & $281 \pm 5$ & $283 \pm 6$ & $280 \pm 5$ & $279 \pm 4$ & $277 \pm 5$ & 0.836 \\
\hline RDW (\%) & $15.5 \pm 0.7$ & $15.7 \pm 0.7$ & $15.8 \pm 0.1$ & $16.1 \pm 0.5$ & $16.1 \pm 0.3$ & $16.2 \pm 0.3$ & $16.5 \pm 0.5$ & 0.804 \\
\hline PLT $\left(10^{9} / \mathrm{L}\right)$ & $360 \pm 40$ & $520 \pm 20$ & $530 \pm 20$ & $520 \pm 40$ & $510 \pm 50$ & $510 \pm 40$ & $500 \pm 30$ & 0.086 \\
\hline MPV (fL) & $7.4 \pm 0.2$ & $7.6 \pm 0.4$ & $8.2 \pm 0.7$ & $7.1 \pm 0.4$ & $7.2 \pm 0.2$ & $6.6 \pm 0.6$ & $7.00 \pm 0.06$ & 0.274 \\
\hline PDW (fL) & $18.5 \pm 0.4$ & $18.0 \pm 0.5$ & $18 \pm 2$ & $17.8 \pm 0.2$ & $18.0 \pm 0.4$ & $17.6 \pm 0.6$ & $17.9 \pm 0.5$ & 0.987 \\
\hline PCT (\%) & $0.27 \pm 0.06$ & $0.33 \pm 0.05$ & $0.31 \pm 0.09$ & $0.22 \pm 0.06$ & $0.21 \pm 0.02$ & $0.24 \pm 0.02$ & $0.19 \pm 0.08$ & 0.640 \\
\hline
\end{tabular}

See Glossary, for definitions of the parameters in the first column. The mean values of rows with the different superscript $(a, b, c)$ are significantly different from each other ( $p<0.05)$. Letter $a b$ indicates that the value is not obviously different from either a or $b$. Letter bc indicates that the value is not obviously different from either $b$ or $c$. Letter abc indicates that the value is not obviously different from either $a, b$, or $c$.

biochemical indicators of liver and kidney function, electrolyte levels, myocardial enzymes, antioxidant levels, glucose metabolism, and lipid metabolism.

Table 2 shows changes in blood cell and hemoglobin parameters after the injection of different PALs into the bone marrow. We observed that the number of white blood cells (WBC), lymphocytes (Lymph), monocytes (Mon), red blood cells (RBC), the percentage of mononuclear cells (Mon\%), hemoglobin (HGB), hematocritg $(\mathrm{HCT})$, mean red blood cell volume (MCV), mean erythrocyte hemoglobin content $(\mathrm{MCH})$, mean erythrocyte hemoglobin concentration (MCHC), erythrocyte distribution width variation coefficient (RDW), mean platelet volume (MPV), platelet distribution width (PDW), and platelet backlog (PCT) were not affected by the different PAL treatments.

There were significant differences in the number of neutrophil granulocytes (Gran) among the groups; however, the values for 
TABLE 3 | Analysis of liver function after different PAL treatments.

\begin{tabular}{|c|c|c|c|c|c|c|c|c|}
\hline Parameter & Con & $\mathbf{J}-\mathbf{N}$ & $\mathbf{J}-\mathbf{M}$ & J-P & $\mathbf{S}-\mathbf{N}$ & S-M & S-P & $p$ Value \\
\hline ALT (U/L) & $60 \pm 10$ & $45 \pm 9$ & $46 \pm 3$ & $61 \pm 6$ & $53 \pm 5$ & $54 \pm 4$ & $58 \pm 8$ & 0.661 \\
\hline AST (U/L) & $50 \pm 10$ & $48 \pm 8$ & $47 \pm 7$ & $51 \pm 8$ & $54 \pm 4$ & $50 \pm 4$ & $52 \pm 1$ & 0.988 \\
\hline ALB (g/L) & $25 \pm 2$ & $24.2 \pm 0.4$ & $24 \pm 1$ & $23.8 \pm 0.8$ & $24.0 \pm 0.5$ & $24.5 \pm 0.5$ & $24 \pm 1$ & 0.933 \\
\hline T-BIL ( $\mu \mathrm{M} / \mathrm{L})$ & $10.1 \pm 0.6$ & $10 \pm 2$ & $9.3 \pm 0.6$ & $9.3 \pm 0.4$ & $9.9 \pm 0.3$ & $8 \pm 1$ & $8.5 \pm 0.2$ & 0.606 \\
\hline D-BIL ( $\mu \mathrm{M} / \mathrm{L})$ & $10 \pm 2$ & $10 \pm 2$ & $10 \pm 1$ & $10.1 \pm 0.1$ & $9.7 \pm 0.2$ & $10 \pm 2$ & $8.8 \pm 0.5$ & 0.997 \\
\hline ALP (U/L) & $60 \pm 10$ & $67 \pm 9$ & $67 \pm 9$ & $70 \pm 10$ & $70 \pm 10$ & $68 \pm 3$ & $65 \pm 8$ & 0.949 \\
\hline
\end{tabular}

See Glossary, for definitions of the parameters in the first column.

\begin{tabular}{|c|c|c|c|c|c|c|c|c|}
\hline Parameter & Con & $\mathrm{J}-\mathrm{N}$ & J-M & J-P & S-N & S-M & S-P & p Value \\
\hline BUN (mg/dl) & $15.4 \pm 0.1$ & $16 \pm 1$ & $16.7 \pm 0.6$ & $15.5 \pm 0.9$ & $16 \pm 2$ & $15.5 \pm 0.5$ & $17 \pm 3$ & 0.968 \\
\hline UA ( $\mu \mathrm{M} / \mathrm{L})$ & $160 \pm 20$ & $140 \pm 30$ & $140 \pm 30$ & $119 \pm 4$ & $140 \pm 20$ & $140 \pm 30$ & $140 \pm 20$ & 0.962 \\
\hline $\mathrm{CR}(\mu \mathrm{M} / \mathrm{L})$ & $90 \pm 10$ & $91 \pm 5$ & $92 \pm 2$ & $87 \pm 9$ & $91 \pm 5$ & $94 \pm 2$ & $90 \pm 5$ & 0.976 \\
\hline $\mathrm{Zn}(\mu \mathrm{M} / \mathrm{L})$ & $13.9 \pm 0.7$ & $14.1 \pm 0.2$ & $14.4 \pm 0.4$ & $13.7 \pm 0.5$ & $14.1 \pm 0.5$ & $13.4 \pm 0.5$ & $14.7 \pm 0.6$ & 0.599 \\
\hline $\mathrm{Fe}(\mu \mathrm{M} / \mathrm{L})$ & $41 \pm 5$ & $42 \pm 1$ & $40.3 \pm 0.7$ & $41 \pm 4$ & $40 \pm 4$ & $41 \pm 6$ & $41 \pm 3$ & 1.000 \\
\hline $\mathrm{Mg}(\mathrm{mM} / \mathrm{L})$ & $1.05 \pm 0.04$ & $1.11 \pm 0.01$ & $1.12 \pm 0.07$ & $1.03 \pm 0.02$ & $1.06 \pm 0.02$ & $1.08 \pm 0.05$ & $1.08 \pm 0.01$ & 0.605 \\
\hline $\mathrm{Cl}(\mathrm{mM} / \mathrm{L})$ & $101 \pm 2$ & $100 \pm 2$ & $105 \pm 3$ & $98 \pm 4$ & $99 \pm 2$ & $105 \pm 4$ & $101 \pm 3$ & 0.561 \\
\hline $\mathrm{p}(\mathrm{mM} / \mathrm{L})$ & $2.0 \pm 0.2$ & $2.00 \pm 0.08$ & $2.0 \pm 0.3$ & $2.04 \pm 0.05$ & $2.0 \pm 0.2$ & $2.1 \pm 0.2$ & $1.99 \pm 0.02$ & 0.999 \\
\hline $\mathrm{Ca}(\mathrm{mM} / \mathrm{L})$ & $2.6 \pm 0.1$ & $2.5 \pm 0.2$ & $2.4 \pm 0.4$ & $2.5 \pm 0.2$ & $2.52 \pm 0.09$ & $2.6 \pm 0.1$ & $2.4 \pm 0.1$ & 0.959 \\
\hline
\end{tabular}

See Glossary, for definitions of the parameters in the first column.

each group were within the normal range (2.0-7.5). In addition, the percentage of neutrophil granulocytes (Gran\%) and lymphocytes (Lymph\%) were clearly affected by the PAL treatments. After performing a post-hoc multiple comparisons or independent sample $t$-test, we found that the Lymph\% in the control group was significantly higher than in the JP, SN, SM, and SP groups. The Gran\% in the control group was clearly lower than in the JM, JP, SN, SM, and SP groups. The platelet count (PLT) tended to be different among the groups $(p=0.086)$, and it was found that PLT was most notably elevated in all PALs with respect to the control group, although they were all within the normal range (100-712).

Alanine transaminase (ALT), aspartate transaminase (AST), albumin (ALB), total bilirubin (T-BIL), direct bilirubin (D-BIL), and alkaline phosphatase (ALP), which are indicators of liver health, did not differ between the different PAL treatments and control group at day 30, indicating that injection of PALs into bone marrow did not cause liver damage (Table 3 ).

Table 4 shows that different PAL treatments did not modify the concentration of nephrotoxicity markers [blood urea nitrogen (BUN), uric acid (UA), creatinine (CR)] and electrolytes (Zn, Fe, $\mathrm{Mg}, \mathrm{Cl}, \mathrm{P}$, and $\mathrm{Ca}$ ) when compared to the control group. Therefore, the different PAL injections were not harmful to kidney function and did not interfere with electrolyte balance.

Furthermore, we analyzed myocardial enzyme levels and antioxidant index after different PAL treatments. The analysis showed that different types of PAL did not affect the myocardial enzymes lactate dehydrogenase (LDH) and creatine kinase (CK), nor the antioxidant indexes superoxide dismutase (SOD), malondialdehyde (MAD), and glutathione peroxidase (GPX)
(Table 5, all $p$ values $>0.05$ ). These results indicate that different PAL treatments do not cause myocardial damage or systemic oxidative stress.

Finally, we investigated serum lipid and glucose metabolism after the different PAL injections into the rabbits. The results did not reveal any significant changes in the levels of triglyceride (TG), high-density lipoprotein cholesterol (HDL-C), low-density lipoprotein cholesterol (LDL-C), glycated serum protein (GSP) or glucose (GLU) between the control group and different PAL treatments (Table 6). It can be inferred therefore that the PAL treatments did not cause lipid or glucose metabolism disorders.

\section{DISCUSSION}

PALs containing various active substances are an area of intense research in the biomedical field, especially in cancer therapy. It has been shown that atmospheric-pressure cold plasma selectively inhibits the growth of cancer cells and causes relatively less or no cytotoxicity in normal cells [10]. As a promising novel approach to cure certain cancers, studies of PAL safety, in assessing the potential side effects, are a basic premise, and it could be classified into three stages: pre-clinical trials, clinical trials in vivo, and detection trials of adverse reactions after approval [21]; the present experiments represent a non-clinical study of PALs. A review of PAL safety studies concluded that up to $25 \%$ of toxic reactions observed in animal experiments may occur in humans [22]. Therefore, we initiated the current study to test the safety of PALs and to provide a basis for clinical research and applications. 
TABLE 5 | Myocardial enzyme and antioxidant index after different PAL treatments.

\begin{tabular}{|c|c|c|c|c|c|c|c|c|}
\hline Parameter & Con & $\mathrm{J}-\mathrm{N}$ & J-M & $J-P$ & S-N & S-M & S-P & $p$ Value \\
\hline LDH (U/L) & $600 \pm 100$ & $700 \pm 100$ & $600 \pm 200$ & $700 \pm 200$ & $730 \pm 30$ & $680 \pm 90$ & $700 \pm 200$ & 0.999 \\
\hline CK (U/L) & $1,600 \pm 900$ & $950 \pm 30$ & $960 \pm 80$ & $1,000 \pm 300$ & $1,000 \pm 100$ & $1,000 \pm 300$ & $1,000 \pm 100$ & 0.887 \\
\hline $\mathrm{SOD}(\mathrm{U} / \mathrm{mL})$ & $300 \pm 20$ & $320 \pm 3$ & $320 \pm 40$ & $330 \pm 20$ & $330 \pm 20$ & $340 \pm 30$ & $340 \pm 20$ & 0.913 \\
\hline MDA (nM/ml) & $5.1 \pm 0.6$ & $4 \pm 1$ & $4.4 \pm 1.0$ & $4.5 \pm 0.8$ & $4.6 \pm 0.2$ & $4.4 \pm 1.0$ & $4.8 \pm 0.5$ & 0.995 \\
\hline GPX (U) & $220 \pm 10$ & $230 \pm 20$ & $240 \pm 20$ & $226 \pm 9$ & $230 \pm 10$ & $250 \pm 10$ & $250 \pm 20$ & 0.857 \\
\hline
\end{tabular}

See Glossary, for definitions of the parameters in the first column.

\begin{tabular}{|c|c|c|c|c|c|c|c|c|}
\hline Parameter & Con & $\mathbf{J}-\mathbf{N}$ & $\mathbf{J}-\mathbf{M}$ & J-P & S-N & S-M & S-P & $p$ Value \\
\hline $\mathrm{TG}(\mathrm{mM} / \mathrm{L})$ & $1.7 \pm 0.2$ & $1.5 \pm 0.1$ & $1.49 \pm 0.04$ & $1.52 \pm 0.04$ & $1.52 \pm 0.09$ & $1.54 \pm 0.08$ & $1.57 \pm 0.08$ & 0.772 \\
\hline HDL-C (mM/L) & $0.5 \pm 0.1$ & $0.49 \pm 0.06$ & $0.48 \pm 0.04$ & $0.52 \pm 0.01$ & $0.5 \pm 0.2$ & $0.5 \pm 0.1$ & $0.5 \pm 0.1$ & 0.999 \\
\hline LDL-C (mM/L) & $3.3 \pm 0.1$ & $2.6 \pm 0.3$ & $2.7 \pm 0.5$ & $2.6 \pm 0.8$ & $2.4 \pm 0.3$ & $2.6 \pm 0.3$ & $2.4 \pm 0.3$ & 0.857 \\
\hline GSP(mM/L) & $2.1 \pm 0.2$ & $2.2 \pm 0.3$ & $2.2 \pm 0.2$ & $1.8 \pm 0.1$ & $2.1 \pm 0.1$ & $2.2 \pm 0.3$ & $2.2 \pm 0.4$ & 0.903 \\
\hline GLU (mM/L) & $6.7 \pm 0.8$ & $7.1 \pm 0.1$ & $8 \pm 1$ & $8 \pm 2$ & $8.7 \pm 0.9$ & $11 \pm 3$ & $7.4 \pm 0.2$ & 0.530 \\
\hline
\end{tabular}

See Glossary, for definitions of the parameters in the first column.

As mentioned in the literature, saline, cell culture medium, and PBS have been widely used in plasma-activated fluids since culture medium is suited to the study of cancer cells while buffers like saline and PBS have a relatively simple composition [23-26]. Hence, we chose them as plasma-activated liquids in the present work. We analyzed changes in the physicochemical properties of the different solutions after plasma jet/surface treatment and found that different PALs had different $\mathrm{pH}$ and ORP values. The PBS used in this study contained a large amount of potassium hydrogen phosphate (weak acid, $\mathrm{KH}_{2} \mathrm{PO}_{4}, \mathrm{pH}$ 4.4-4.9) and sodium hydrogen phosphate (weak base, $\mathrm{Na}_{2} \mathrm{HPO}_{4}, \mathrm{pH}$ 9.5) that makes it a strong buffer, so the $\mathrm{pH}$ and ORP of PBS did not change after the jet treatment when compared with the control group (Figures 3A,B). Jirasek et al. [27] reported that $85 \%$ hypochlorite in a physiological $\mathrm{NaCl}$ solution treated by a plasma jet existed in the form of $\mathrm{NaOCl}$, and as a consequent had a high $\mathrm{pH}$, consistent with our results (Figure 3A). As RPMI 1640 medium includes polysaccharides, amino acids, vitamins, and many other compounds, the increased $\mathrm{pH}$ of the jet-treated culture medium might be due to reaction of these organic compounds with plasma-generated chemicals (particularly ROS and RNS). Moreover, because of the disparate discharge gases of these two plasma devices and the greater variety of gases in the plasma surface discharge, the rapid decrease of $\mathrm{pH}$ in the three kinds of surface-treated solutions might be caused by reaction of the vapor-phase $\mathrm{NO}_{x}$ species with $\mathrm{H}_{2} \mathrm{O}$ molecules to produce $\mathrm{HNO}_{3}$, resulting in the generation of a large number of $\mathrm{H}^{+}$ions $[28,29]$. The $\mathrm{pH}$ of 1,640 medium and PBS after plasma surface treatment did not decrease as significantly as that of saline solution, which was due to the buffering ability of 1,640 medium and PBS. ORP, a concentration-dependent indicator of solution oxidation capacity, is commonly used to evaluate the global level of RONS in PALs. Changes in ORP are often opposite to those in $\mathrm{pH}$, which may result from PALs having a greater oxidizing capacity at lower $\mathrm{pH}[30]$.

In this study, the plasma jet was used with $\mathrm{He}+0.5 \% \mathrm{O} 2$ and the plasma surface with ambient air, which are considered the most commonly used methods of applying plasma [10, 31]. Our previous results showed that $3 \mathrm{~min}$ of PAL treatment with either device could inhibit the growth of cancer cells since they generate various kinds of ROS and RNS, like hydroxyl radical $(\mathrm{OH})$, hydrogen peroxide $\left(\mathrm{H}_{2} \mathrm{O}_{2}\right)$, atomic oxygen $(\mathrm{O})$, nitric oxide $(\mathrm{NO})$, and peroxynitrite anion $\left(\mathrm{ONOO}^{-}\right)$(Figure 4) [10, 31]. Figure 5 shows the active particles in the gaseous state, and prior studies noted that only small amounts of $\mathrm{O}_{3}, \mathrm{H}_{2} \mathrm{O}_{2}, \mathrm{OH}$, and $\mathrm{NO}_{\mathrm{x}}$ could pass through the gas-liquid interface $[23,24]$, while other ROS/RNS are transferred into liquid via secondary reactions [32]. Unlike $\mathrm{H}_{2} \mathrm{O}_{2}$, which can penetrate the cell membrane, most other radicals cannot and so may play roles in cell-signal transduction [10]. Furthermore, the addition of $0.5 \%$ O2 generated more ROS and caused more cell death compared with He plasma alone [10]. The generation pathway of $\mathrm{H}_{2} \mathrm{O}_{2}$ is mainly due to the combination reaction of aqueous hydroxide radical (.OH) [33]. As shown in Figure 5, bands of $\mathrm{OH}$ radicals were observed in the spectra of three different liquids treated by the plasma jet device, but not in the plasma surface device. Therefore, the concentration of $\mathrm{H}_{2} \mathrm{O}_{2}$ produced by the jet was much higher than that produced by the surface device (Figure 4B). Bands of $\mathrm{N}$ excitation were found in both devices for treating three different liquids (Figure 5), and they had significant effects on the production of $\mathrm{NO}_{2}$ and $\mathrm{NO}_{3}$.

$\mathrm{PAL}$ is administered via intravenous, intra-arterial, intramuscular, oral, intra-luminal injection, and other routes $[34,35]$. In the present experiments, we adopted the route of intramedullary injection. Firstly, intramedullary injection is suitable for myeloma and blood tumors, especially since pathogenesis is in the bone marrow cavity, and the drug can 
directly act on the tumor site without being affected by the bone marrow-blood barrier. Likewise, many stem cells exist in bone marrow, and the current study provides a basis for further treatment of tumors by intramedullary injection of PAL to kill tumor stem cells [36]. Lastly, the advantage of intramedullary injection is that it can induce both local and systemic effects. Additionally, Wei Zhang et al. [37] found both intravenous and paravertebral dexmedetomidine attenuated independent lung injury.

Generally, we chose a higher dose of material for the acute toxicity test, and our results showed that rabbits injected with $700 \mu \mathrm{L}$ of PAL did not exhibit any mortality, loss of mobility, or visible signs of toxicity when compared to the control group during the 30-days experimental period.

Changes in body and organ weight are sensitive indicators of the general health of animals [38]. Studies show that a loss of body weight of more than $20 \%$ should be considered a serious condition that can also impact organ size and weight [39-41]. Thus, the organ-to-body weight index is regarded as a useful indicator of the toxicity of new compounds. No morbidity or mortality was evident throughout the experimental period, and body weight of the rabbits actually increased similarly in the control and PAL-treated groups by day 30 (Figure 6A). Furthermore, in comparison with the control group, PAL treatment caused no lesions in the heart, liver, spleen, lung, or kidney (Figure 6C), and there were no significant differences in organ index (Figure 6B). Thus, we conclude that the different PALs had no adverse effects on growth of the rabbits.

Changes in histopathology of organs, examined by microscopy, are also considered a basic test of the safety of the materials [42]. No abnormality was observed in histopathological sections of the organs in this study (Figure 7), indicating that the different PALs did not induce any toxic symptoms in the major internal organs, including the heart, liver, lung, and kidney.

Hematological analyses are of critical importance in the evaluation of toxic effects induced by materials [43]. Due to blood being the main transporter of many xenobiotics in the body, blood components are exposed to numerous toxic compounds that induce damage or destruction of blood cells and harm normal physiological function [44, 45]. Our analyses of blood biochemistry in the treated rabbits suggested that the three types of PAL did not alter normal liver/hepatic/ myocardial function, oxidative stress, and lipid or glucose metabolism. Observations of these blood parameters were consistent with measurements of body weight, organ index, organ appearance, and organ histopathology, further validating the absence of toxicity in response to PALs. Furthermore, our observations of blood parameters normally associated with systemic toxic symptoms showed that most were not affected by PAL treatment. In addition, the increase in the PLT count after PAL treatment revealed that PAL might accelerate blood clotting, as PLTs are small pieces of cytoplasm that detach from the cytoplasm of mature megakaryocytes of the bone marrow and are essential for hemostasis [46]. The findings of other studies also suggest that platelets are important for cold physical plasma-facilitated blood coagulation [47, 48]. However, Gran\% increased and
Lymph\% decreased after PAL treatment, suggesting that PAL treatment might improve the activity of the immune system. Shi et al. [49] took peripheral blood lymphocytes from 20 healthy adults and found that plasma could induce apoptosis of lymphocytes as evidenced by leakage of protein, nucleic acid, and $\mathrm{K}^{+}$after treatment, consistent with our observations of lymphocytes. It is worth noting that different immune cells exhibit distinct sensitivities after plasma treatment [50,51]. Bundscherer et al. [51] observed that the number of $\mathrm{CD}^{+} \mathrm{T}$ helper cells, a kind of lymphocyte, decreased significantly in a time-dependent manner after $60 \mathrm{~s}$ of plasma treatment and there was a marked attenuation in monocyte growth after exposure to plasma for $360 \mathrm{~s}$. In our study, $3 \mathrm{~min}$ of plasma treatment reduced Lymph\% but did not affect Mon\%, which was similar to the results of Bundscherer et al. [51]. Therefore, we speculate that the different changes in the levels of lymphocytes and monocytes after plasma treatment may be due to the duration of plasma treatment and the distinct sensitivity of immune cells to plasma. Meanwhile, as white blood cell count was not significantly different among groups, we suggest the increase of Gran\% may be due to the reduction of Lymph\%. Moreover, Lardner A (2001) reported that extracellular $\mathrm{pH}$ affected immune cells and immune function [52], so we suggested that the different $\mathrm{pH}$ values of PALs (Figure 3) might contribute to the changes of Gran\% and Lymph\% (Table 2).

Although the physicochemical properties and the active substances varied among the three PALs in our study, PAL injection did not cause toxicity, indicating that PAL treatment is safe for New Zealand rabbits when injected into the bone marrow, and therefore has great potential for development of clinical therapeutics. In the future, we will evaluate the safety and efficacy of PAL in humans and principally focus on clinical application of PALs in hematologic tumors such as leukemia and multiple myeloma. Specifically, PAL will be used alone or in association with other conventional chemotherapeutic agents to kill cancer cells and stem cells within the marrow cavities of patients during myeloablative treatments prior to bone marrow transplantation.

\section{CONCLUSION}

This work provides a foundation for safety and toxicity studies of PALs. PAL treatment of New Zealand rabbits by intra-bone marrow injection was not lethal and did not lead to any acute toxicity. In addition, PAL treatments did not affect body weight or the coefficient, appearance, or histopathology of the major organs. These results were consistent with blood biochemical indexes showing that PAL treatment had no observable effects on liver/renal/myocardial function, oxidative status, electrolyte and glucose levels, or lipid metabolism. Furthermore, PAL treatment decreased the percentage of lymphocytes while increasing the percentage of neutrophil granulocytes. In conclusion, the results of the present study indicate that PAL treatment can be used safely 
on New Zealand rabbits without significant toxicity when injected into bone marrow.

\section{DATA AVAILABILITY STATEMENT}

The original contributions presented in the study are included in the article/supplementary material, further inquiries can be directed to the corresponding authors.

\section{ETHICS STATEMENT}

The animal study was reviewed and approved by the Experimental Animal Center of Xi'an Jiaotong University which has obtained the experimental animal production license [SCXK (shaan) 2008-008] and animal use certificate [SYXK (shaan) 2008-002] issued by the Science and Technology department of Shaanxi province.

\section{REFERENCES}

1. Global cancer Observatory. Cancer today. https://gco.iarc.fr/today/home.

2. Leary M, Heerboth S, Lapinska K, and Sarkar S. Sensitization of Drug Resistant Cancer Cells: A Matter of Combination Therapy. Cancers (2018) 10:483. doi:10.3390/cancers10120483

3. Friberg S, and Nyström A. Cancer Metastases: Early Dissemination and Late Recurrences. Cancer Growth Metastasis (2015) 8:43-9. doi:10.4137/ CGM.S31244

4. Graves D, Hamaguchi S, and O'Connell D. In Focus: Plasma Medicine. Biointerphases (2015) 10:029301. doi:10.1116/1.4922357

5. Shome D, von Woedtke T, Riedel K, and Masur K. The HIPPO Transducer YAP and its Targets CTGF and Cyr61 Drive a Paracrine Signalling in Cold Atmospheric Plasma-Mediated Wound Healing. Oxidative Med Cell Longevity (2020) 2020:1-14. doi:10.1155/2020/4910280

6. Hoffmann C, Berganza C, and Zhang J. Cold Atmospheric Plasma: methods of production and application in dentistry and oncology. Med Gas Res (2013) 3: 21-15. doi:10.1186/2045-9912-3-21

7. Lou B-S, Lai C-H, Chu T-P, Hsieh J-H, Chen C-M, Su Y-M, et al. Parameters Affecting the Antimicrobial Properties of Cold Atmospheric Plasma Jet. Jcm (2019) 8:1930. doi:10.3390/jcm8111930

8. Heinlin J, Isbary G, Stolz W, Morfill G, Landthaler M, Shimizu T, et al. Plasma applications in medicine with a special focus on dermatology. J Eur Acad Dermatol Venereol (2011) 25:1-11. doi:10.1111/j.1468-3083.2010.03702.x

9. Thiyagarajan M, Waldbeser L, and Whitmill A. THP-1 leukemia cancer treatment using a portable plasma device. Stud Health Technol Inform (2012) 173:515-7.

10. Xu D, Xu Y, Cui Q, Liu D, Liu Z, Wang X, et al. Cold atmospheric plasma as a potential tool for multiple myeloma treatment. Oncotarget (2018) 9:18002-17. doi:10.18632/oncotarget. 24649

11. Schneider C, Arndt S, Zimmermann JL, Li Y, Karrer S, and Bosserhoff AK. Cold atmospheric plasma treatment inhibits growth in colorectal cancer cells. Biol Chem (2018) 400:111-22. doi:10.1515/hsz-2018-0193

12. Schneider C, Gebhardt L, Arndt S, Karrer S, Zimmermann JL, Fischer MJM, et al. Acidification is an Essential Process of Cold Atmospheric Plasma and Promotes the Anti-cancer Effect on Malignant Melanoma Cells. Cancers (2019) 11:671. doi:10.3390/cancers11050671

13. Griseti E, Merbahi N, and Golzio M. Anti-Cancer Potential of Two PlasmaActivated Liquids: Implication of Long-Lived Reactive Oxygen and Nitrogen Species. Cancers (2020) 12:721. doi:10.3390/cancers12030721

14. Hirst AM, Frame FM, Arya M, Maitland NJ, and O'Connell D. Low temperature plasmas as emerging cancer therapeutics: the state of play and thoughts for the future. Tumor Biol (2016) 37:7021-31. doi:10.1007/s13277016-4911-7

\section{AUTHOR CONTRIBUTIONS}

YX and SP performed the experiments and writing the manuscript; DX and DL conceived and supervised the study; $\mathrm{BL}, \mathrm{SW}$, and QL participated in the experiment work; HZ, ZL, and $\mathrm{BG}$ contributed to the figures of this study. All authors have read and agreed to the published version of the manuscript.

\section{FUNDING}

This work is supported by the National Natural Science Foundation of China (Grant No. 51521065 and 51837008), China Postdoctoral Science Foundation (2017M610639) and Special Fund of China Postdoctoral Science Foundation, State Key Laboratory of Electrical Insulation and Power Equipment (EIPE19309) and Special Fund of Shaanxi Postdoctoral Science Foundation (2017BSHTDZZ04).

15. Xu D, Liu D, Wang B, Chen C, Chen Z, Li D, et al. In Situ OH Generation from O2- and H2O2 Plays a Critical Role in Plasma-Induced Cell Death. PLoS One (2015) 10:e0128205. doi:10.1371/journal.pone.0128205

16. Judée F, Fongia C, Ducommun B, Yousfi M, Lobjois V, and Merbahi N. Short and long time effects of low temperature Plasma Activated Media on 3D multicellular tumor spheroids. Sci Rep (2016) 6:21421. doi:10.1038/srep21421

17. Adachi T, Tanaka H, Nonomura S, Hara H, Kondo S-i., and Hori M. Plasmaactivated medium induces A549 cell injury via a spiral apoptotic cascade involving the mitochondrial-nuclear network. Free Radic Biol Med (2015) 79: 28-44. doi:10.1016/j.freeradbiomed.2014.11.014

18. Yan D, Cui H, Zhu W, Nourmohammadi N, Milberg J, Zhang LG, et al. The Specific Vulnerabilities of Cancer Cells to the Cold Atmospheric PlasmaStimulated Solutions. Sci Rep (2017) 7:4479. doi:10.1038/s41598-017-04770-x

19. Canal C, Fontelo R, Hamouda I, Guillem-Marti J, Cvelbar U, and Ginebra M-P. Plasma-induced selectivity in bone cancer cells death. Free Radic Biol Med (2017) 110:72-80. doi:10.1016/j.freeradbiomed.2017.05.023

20. Biscop E, Lin A, Boxem WV, Loenhout JV, Backer JD, Deben C, et al. Influence of Cell Type and Culture Medium on Determining Cancer Selectivity of Cold Atmospheric Plasma Treatment. Cancers (2019) 11:1287. doi:10.3390/ cancers11091287

21. Yao B, Zhu L, Jiang Q, and Xia H. Safety monitoring in clinical trials. Pharmaceutics (2013) 5:94-106. doi:10.3390/pharmaceutics5010094

22. Fletcher AP. Drug safety tests and subsequent clinical experience. J R Soc Med (1978) 71:693-6. doi:10.1177/014107687807100915

23. Bauer G. The synergistic effect between hydrogen peroxide and nitrite, two long-lived molecular species from cold atmospheric plasma, triggers tumor cells to induce their own cell death. Redox Biol (2019) 26:101291. doi:10.1016/ j.redox.2019.101291

24. Van Boxem W, Van der Paal J, Gorbanev Y, Vanuytsel S, Smits E, Dewilde S, et al. Anti-cancer capacity of plasma-treated PBS: effect of chemical composition on cancer cell cytotoxicity. Sci Rep (2017) 7:16478. doi:10.1038/s41598-017-16758-8

25. Mateu-Sanz M, Tornín J, Brulin B, Khlyustova A, Ginebra M-P, Layrolle P, et al. Cold Plasma-Treated Ringer's Saline: A Weapon to Target Osteosarcoma. Cancers (2020) 12:227. doi:10.3390/cancers12010227

26. Tanaka H, Mizuno M, Katsumata $Y$, Ishikawa K, Kondo H, Hashizume H, et al. Oxidative stress-dependent and -independent death of glioblastoma cells induced by non-thermal plasma-exposed solutions. Sci Rep (2019) 9:13657. doi:10.1038/s41598-019-50136-w

27. Jirásek $\mathrm{V}$, and Lukeš $\mathrm{P}$. Formation of reactive chlorine species in saline solution treated by non-equilibrium atmospheric pressure $\mathrm{He} / \mathrm{O} 2$ plasma jet. Plasma Sourc Sci. Technol. (2019) 28:035015. doi:10.1088/1361-6595/ab0930

28. Lukes P, Dolezalova E, Sisrova I, and Clupek M. Aqueous-phase chemistry and bactericidal effects from an air discharge plasma in contact with water: evidence for the formation of peroxynitrite through a pseudo-second-order 
post-discharge reaction of H2O2and HNO2. Plasma Sourc Sci. Technol. (2014) 23:015019. doi:10.1088/0963-0252/23/1/015019

29. Zhang X, Zhou R, Zhou R, Chen M, Li J, Sun Y, et al. Treatment of Ribonucleoside Solution with Atmospheric-Pressure Plasma. Plasma Process Polym (2016) 13:429-37. doi:10.1002/ppap.201500088

30. Zhou R, Zhou R, Prasad K, Fang Z, Speight R, Bazaka K, et al. Cold atmospheric plasma activated water as a prospective disinfectant: the crucial role of peroxynitrite. Green Chem (2018) 20:5276-84. doi:10.1039/ c8gc02800a

31. Zhong SY, Dong YY, Liu DX, Xu DH, Xiao SX, Chen HL, et al. Surface air plasma-induced cell death and cytokine release of human keratinocytes in the context of psoriasis. Br J Dermatol (2016) 174:542-52. doi:10.1111/bjd.14236

32. Tornin J, Mateu-Sanz M, Rodríguez A, Labay C, Rodríguez R, and Canal C. Pyruvate Plays a Main Role in the Antitumoral Selectivity of Cold Atmospheric Plasma in Osteosarcoma. Sci Rep (2019) 9:10681. doi:10.1038/s41598-01947128-1

33. Chen Z, Liu D, Chen C, Xu D, Liu Z, Xia W, et al. Analysis of the production mechanism of $\mathrm{H} 2 \mathrm{O} 2$ in water treated by helium DC plasma jets. J Phys D: Appl Phys (2018) 51:325201. doi:10.1088/1361-6463/aad0eb

34. Shim J, Kwak BK, Jung J, and Park S. Evaluation of engraftment of superparamagnetic iron oxide-labeled mesenchymal stem cells using threedimensional reconstruction of magnetic resonance imaging in photothrombotic cerebral infarction models of rats. Korean J Radiol (2015) 16:575-85. doi:10.3348/kjr.2015.16.3.575

35. Shi Y, Peng D, Wang D, Zhao Z, Chen B, He B, et al. Biodistribution Survey of Oxidized Single-Wall Carbon Nanohorns Following Different Administration Routes by Using Label-free Multispectral Optoacoustic Tomography. Ijn (2019) Vol. 14:9809-21. doi:10.2147/ijn.s215648

36. Feng R, Ning N, Tian M, Peng S, Wang S, Li B, et al. Evaluation of the anticancer effects induced by cold atmospheric plasma on leukemia stem cells. Plasma Res Express (2020) 2:024001. doi:10.1088/2516-1067/ab9154

37. Zhang W, Zhang S, Li B, Sun M, and Zhang J. Paravertebral dexmedetomidine as an adjuvant to ropivacaine protects against independent lung injury during one-lung ventilation: a preliminary randomized clinical trial. BMC Anesthesiol (2018) 18:67. doi:10.1186/s12871-018-0532-6

38. Chitra B, Ramaswamy RS, and Suba V. Toxicity Evaluation of Pũrna Cantirotaya Centũram, a Siddha Medicine in Wistar Rats. Int scholarly Res notices (2015) 2015:1-10. doi:10.1155/2015/473296

39. William S. Guidance Document on the Recognition, Assessment, and Use of Clinical Signs as Humane Endpoints for Experimental Animals Used in Safety Evaluations 2000.

40. Olfert E, Bhasin J, Latt R, Macallum E, Mccutcheon K, Rainnie D, et al. CCAC Guidelines on: Choosing an Appropriate Endpoint in Experiments Using Animals for Research. Teaching and Testing (1998). Ottawa, Canada: Canadian Council on AnimalCare.

41. Lazic SE, Semenova E, and Williams DP. Determining organ weight toxicity with Bayesian causal models: Improving on the analysis of relative organ weights. Sci Rep (2020) 10:6625. doi:10.1038/s41598-020-63465-y

42. Wolf JC, and Maack G. Evaluating the credibility of histopathology data in environmental endocrine toxicity studies. Environ Toxicol Chem (2017) 36: 601-11. doi:10.1002/etc.3695
43. Petterino C, and Argentino-Storino A. Clinical chemistry and haematology historical data in control Sprague-Dawley rats from pre-clinical toxicity studies. Exp Toxicologic Pathol (2006) 57:213-9. doi:10.1016/j.etp.2005.10.002

44. Abotsi W, Ainooson G, and Boakye-Gyasi E. Acute and Sub-acute Toxicity Studies of the Ethanolic Extract of the Aerial Parts of Hilleria Latifolia (Lam.) H. Walt.(Phytolaccaceae) in Rodents. West Afr J Pharm (2011) 22:27-35.

45. Olson H, Betton G, Robinson D, Thomas K, Monro A, Kolaja G, et al. Concordance of the toxicity of pharmaceuticals in humans and in animals. Regul Toxicol Pharmacol (2000) 32:56-67. doi:10.1006/rtph.2000.1399

46. Baccini V, Geneviève F, Jacqmin H, Chatelain B, Girard S, Wuilleme S, et al. Platelet Counting: Ugly Traps and Good Advice. Proposals from the FrenchSpeaking Cellular Hematology Group (GFHC). Jcm (2020) 9:808. doi:10.3390/ jcm9030808

47. Bekeschus S, Brüggemeier J, Hackbarth C, von Woedtke T, Partecke L-I, and van der Linde J. Platelets are key in cold physical plasma-facilitated blood coagulation in mice. Clin Plasma Med (2017) 7-8:58-65. doi:10.1016/j.cpme.2017.10.001

48. Bekeschus S, Brüggemeier J, Hackbarth C, Weltmann K-D, von Woedtke T, Partecke L-I, et al. The feed gas composition determines the degree of physical plasma-induced platelet activation for blood coagulation. Plasma Sourc Sci. Technol. (2018) 27:034001. doi:10.1088/1361-6595/aaaf0e

49. Shi X, Zhang G, Yuan Y, Ma Y, Xu G, and Yang Y. Effects of low-temperature atmospheric air plasmas on the activity and function of human lymphocytes. Plasma Process Polym (2010) 5:482-8.

50. Haertel B, Volkmann F, von Woedtke T, and Lindequist U. Differential sensitivity of lymphocyte subpopulations to non-thermal atmosphericpressure plasma. Immunobiology (2012) 217:628-33. doi:10.1016/ j.imbio.2011.10.017

51. Bundscherer L, Wende K, Ottmüller K, Barton A, Schmidt A, Bekeschus S, et al. Impact of non-thermal plasma treatment on MAPK signaling pathways of human immune cell lines. Immunobiology (2013) 218:1248-55. doi:10.1016/ j.imbio.2013.04.015

52. Lardner A. The effects of extracellular $\mathrm{pH}$ on immune function. J Leukoc Biol (2001) 69:522-30.

Conflict of Interest: The authors declare that the research was conducted in the absence of any commercial or financial relationships that could be construed as a potential conflict of interest.

Publisher's Note: All claims expressed in this article are solely those of the authors and do not necessarily represent those of their affiliated organizations, or those of the publisher, the editors and the reviewers. Any product that may be evaluated in this article, or claim that may be made by its manufacturer, is not guaranteed or endorsed by the publisher.

Copyright $\odot 2021 \mathrm{Xu}$, Peng, Li, Wang, Zhang, Li, Liu, Guo, Liu and Xu. This is an open-access article distributed under the terms of the Creative Commons Attribution License (CC BY). The use, distribution or reproduction in other forums is permitted, provided the original author(s) and the copyright owner(s) are credited and that the original publication in this journal is cited, in accordance with accepted academic practice. No use, distribution or reproduction is permitted which does not comply with these terms. 


\section{GLOSSARY}

ALB albumin

ALP alkaline phosphatase

ALT alanine transaminase

ANOVA analysis of variance

AST aspartate transaminase

BUN blood urea nitrogen

CAP cold atmospheric plasma

CK creatine kinase

CR creatinine

D-BIL direct bilirubin

GLU glucose

GPX glutathione peroxidase

Gran number of neutrophil granulocyte

Gran\% percentage of neutrophil granulocyte

GSP glycated serum protein

HCT hematocritg

HDL-C high-density lipoprotein cholesterol

HE hematoxylin and eosin

HGB hemoglobin

$\mathbf{H}_{2} \mathbf{O}_{2}$ Hydrogen peroxide

LDH lactate dehydrogenase

LDL-C low-density lipoprotein cholesterol

Lymph number of lymphocytes

Lymph\% percentage of lymphocytes

MAD malondialdehyde

MCH mean erythrocyte hemoglobin content

MCHC mean erythrocyte hemoglobin concentration
MCV mean red blood cell volume

Mon number of monocytes

Mon\% percentage of mononuclear cells

MPV mean platelet volume

MTD maximum tolerated dose

NO nitric oxide

$\mathbf{O}$ atomic oxygen

OCT optimal cutting temperature

OES optical emission spectroscopy

$\mathbf{O H}$ hydroxyl radical

$\mathrm{ONOO}^{-}$peroxynitrite anion.

ORP oxidation-reduction potential

PAL plasma-activated liquid

PBS phosphate buffer solution

PCT platelet backlog

PDW platelet distribution width

PLT platelet count

RBC number of red blood cells

RDW erythrocyte distribution width variation coefficient

RNS nitrogen-based reactive species

RONS reactive oxygen and nitrogen species

ROS oxygen-based reactive species

RPMI Roswell Park Memorial Institute

SEM standard error of the mean

SOD superoxide dismutase

T-BIL total bilirubin

TG triglyceride

UA uric acid

WBC white blood cells 